\section{Mycophenolate mofetil (MMF) following penetrating high- risk keratoplasty: long-term results of a prospective, randomised, multicentre study}

\begin{abstract}
Background The purpose of this prospective, randomised, multicentre study was to prove the efficacy and safety of mycophenolate mofetil (MMF) in preventing graft rejection and in improving clear graft survival following high-risk keratoplasty.

Methods In all, 98 of 140 scheduled patients were included in this study (57 MMF, 41 control). Recruitment was stopped prematurely due to a statistically significant result. The patients in the MMF group received MMF orally $2 \times 1 \mathrm{~g}$ daily for 6 months. All of the patients received fluocortolone $1 \mathrm{mg} / \mathrm{kg} /$ day tapered over 3 weeks and topical prednisolone acetate $5 \times$ /day tapered over 5 months. Main criteria were immune reaction-free and clear graft survival, and the occurrence of side effects. Results The mean follow-up time was $34.9 \pm 16.3$ (mean \pm SD) months. Eleven patients withdrew from the study (nine patients due to protocol deviation, two because of side effects). Six reversible and two irreversible graft rejections occurred in the MMF group, and five reversible and seven irreversible rejections in the control group. The Kaplan-Meier analysis revealed an immune reaction-free graft survival after the mean follow-up time of $83 \%$ in the MMF group and $64.5 \%$ in the control group $(P=0.044)$. Graft failure occurred in $10 \mathrm{MMF}-$ treated patients (two due to rejection) and in nine patients in the control group (seven due to rejection). A total of 36 of 57 MMF-treated
\end{abstract}

F Birnbaum', S Mayweg ${ }^{2}$, A Reis ${ }^{1}$, D Böhringer ${ }^{1}$, B Seitz ${ }^{3}$, K Engelmann ${ }^{4}$, EM Messmer ${ }^{5}$ and $T^{T}$ Reinhard $^{1}$

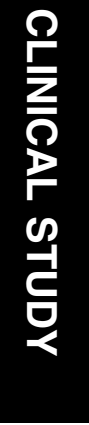

${ }^{1}$ University Eye Hospital, Killianstr 5, Freiburg, Germany

${ }^{2}$ University Eye Hospital, Moorenstr 5, Düsseldorf, Germany

patients experienced mostly reversible adverse events.

Conclusions Systemic immunosuppression with MMF over 6 months is relatively well tolerated and improves rejection-free graft survival following high-risk keratoplasty statistically significant, even in the long run. Eye (2009) 23, 2063-2070; doi:10.1038/eye.2008.402; published online 16 January 2009

Keywords: high-risk keratoplasty; immunosuppression; mycophenolate mofetil

Introduction

Immunological graft rejection following corneal transplantation is the major threat to clear graft survival, especially in penetrating high-risk keratoplasty. ${ }^{1-3}$ Systemic immunosuppression with cyclosporin A (CSA), mycophenolate mofetil (MMF), or rapamycin following penetrating high-risk keratoplasty improved corneal graft prognosis considerably in some prospective and retrospective monocentre studies. ${ }^{4-7}$ Nevertheless, postoperative systemic immunosuppression is still not routinely administered in penetrating high-risk keratoplasty, in contrast to solid organ transplantation. To date, there is no immunosuppressive drug approved for postoperative prophylaxis of corneal transplantation.

This is the first prospective, randomised, multicentre study with the purpose of proving the efficacy of systemic immunosuppression
${ }^{3}$ University Eye Hospital, Kirrberger Straße, Homburg/ Saar, Germany

${ }^{4}$ Eye Hospital, Flemmingstr 2, Chemnitz, Germany

${ }^{5}$ Department of Maximilians-University, Mathildenstr 8, Munich, Germany

Correspondence:

F Birnbaum,

University Eye Hospital

Freiburg,

Freiburg 79106,

Germany

Tel: + 497612704001 ;

Fax: + 497612704063 .

E-mail: florian.birnbaum@ uniklinik-freiburg.de

Received: 7 March 2008 Accepted in revised form: 1 December 2008

Published online: 16 January 2009

This work was presented as free paper during the annual convention of the German Ophthalmological Society (DOG) in Berlin, 21-24 September 2006 Study registration number: NCT00411515; and ZKS UKF000727 (www. uniklinik-freiburg.de/zks/ live/uklregister/ Oeffentlich.html)
Ophthalmology, Ludwig-

Killianstr 5, 
with MMF following high-risk keratoplasty. Results are compared with a control group without mid-term systemic immunosuppression. Preliminary short-term results from this study before patient recruitment completion have already been published. ${ }^{8}$ We now present the final results from this study with long-term follow-up.

MMF (CellCept ${ }^{\circledR}$, Roche Pharma AG, Grenzach-Wyhlen, Germany) is the bioavailabilityenhanced prodrug of the active substance mycophenolic acid (MPA). MPA inhibits the de novo synthesis of guanosine nucleotides by reversibly inhibiting inosine monophosphate dehydrogenase. ${ }^{9-11}$ This leads to selective inhibition of T- and B-lymphocyte proliferation, as they are dependent on de novo purine synthesis. ${ }^{9}$ Other tissues are not, or are less influenced by MPA due to their ability to use the alternative salvage pathway of purine synthesis. In kidney transplantation, MMF is approved at a fixed dose of $2 \times 1000 \mathrm{mg}$ per day in combination with CSA and steroids. ${ }^{12}$ Means of further improving the efficacy of MMF with therapeutic drug monitoring are under investigation. Typical side effects of MPA are infections, leucopaenia, anaemia, and gastrointestinal disturbances possibly due to MPA's mechanism of action. $^{13}$

MMF has been shown to be efficient and safe after kidney, heart, and liver transplantation, ${ }^{12-18}$ and its potency has been proven in preventing corneal allograft rejection in the rat keratoplasty model. ${ }^{19,20} \mathrm{MMF}$ has been widely used in organ transplantation for over 10 years.

\section{Materials and methods}

The study registration number is NCT00411515.

\section{Patient selection, treatment, and follow-up}

A total of nine centres participated in this study. The trial started in June 2000 and the last follow-up examination was performed in August 2006.

A total of 98 of 140 scheduled patients were included in this study. Fifty-seven patients were randomised to receive MMF and 41 patients to undergo no mid-term systemic immunosuppression. Randomisation was performed by drawing a lot. Study recruitment was stopped prematurely due to a statistically significant result from the scheduled interim evaluation.

All patients gave written informed consent, once the approval from the local ethics committees of all participating centres had been obtained.

We defined high-risk keratoplasty as repeat keratoplasty, graft position close to the limbus, presence of three or four quadrants with deep vascularisation, transplantation of a highly immunogenic graft (eg, central limbokeratoplasty $\left.{ }^{21}\right)$, severe atopic dermatitis, and steroid-response glaucoma. ${ }^{3,6,22,23}$ Patient data and the frequencies of the indications in this study are listed in Table 1.

Exclusion criteria were herpetic keratitis or other infectious corneal diseases because of the difficulties in differentiating an intraocular herpes recurrence from an immune reaction, and the risk of triggering an immune reaction due to a herpes recurrence. A complete medical history was taken and physical and laboratory examinations performed for each patient. History of acute or chronic systemic infections, peptic ulcer disease, malignant disorders, inadequate contraceptive measures, pregnancy, or age under 18 years were exclusion criteria for systemic immunosuppression with MMF.

Patients received grafts (preserved in organ culture) having an endothelial cell density of at least 2000 cells $/ \mathrm{mm}^{2}$.

The HLA types of donor and recipient were known at HLA loci A, B, and DR for 23 patients (Table 2). HLA typing was carried out using serological tests for class I and polymerase chain reaction for class II.

Penetrating keratoplasty was performed with a double-running diagonal suture with $2 \times 8$ crossstitches. ${ }^{24}$ Cataract surgery was performed simultaneously in six patients in the MMF group and in two patients in the control group.

The first and the second sutures were not removed until the 4 th and the 18th months, respectively, after keratoplasty. Sutures were removed using topical anaesthesia with proxymetacaine eye drops.

Group 1 patients received MMF (CellCept ${ }^{\circledR}$, Roche Pharma AG, Grenzach-Wyhlen, Germany) at a fixed dosage $(2 \times 1000 \mathrm{mg}$ daily) for 6 months postoperatively. Thereafter, they took $2 \times 500 \mathrm{mg}$ MMF daily for 2 weeks. Blood level monitoring of MMF was not performed. Group 2 patients underwent no mid-term systemic immunosuppression. All patients (groups 1 and 2) received systemic and topical corticosteroids (except the one patient with steroid-response glaucoma):

fluocortolone (Ultralan ${ }^{\circledR}$, Schering Health, Germany) at $1 \mathrm{mg} / \mathrm{kg}$ body weight per day, tapered over 3 weeks, and prednisolone acetate $1 \%$ eye drops (Inflanefran forte ${ }^{\circledR}$, Pharm-Allergan, Germany) at five times a day, tapered over 5 months.

Postoperative examination was performed after 6 weeks, 3, 6, 9, 12, 18, and 24 months, and then yearly, including visual acuity, slit-lamp examination, specular microscopy of the graft endothelium, determination of intraocular pressure, and fundus examination. Adverse events and possible systemic side effects were monitored (in cooperation with each patient's general practitioner) by means of a standard list of questions. 
Table 1 Patient data (mean \pm SD)

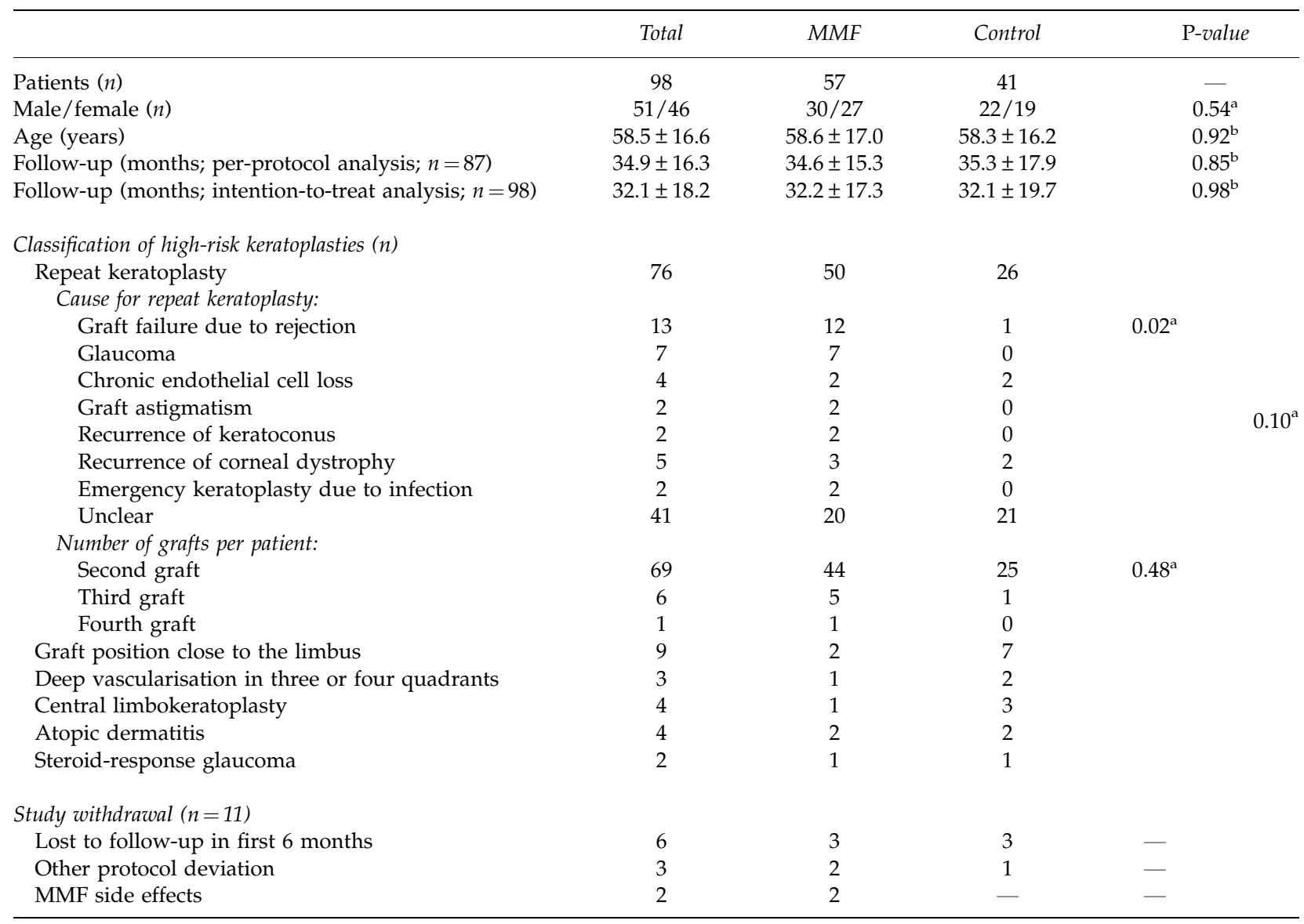

${ }^{\mathrm{a}} \chi^{2}$-test.

${ }^{\mathrm{b} A N O V A .}$

Table 2 Graft data and HLA matching (per-protocol analysis $(n=87$; mean \pm SD)

\begin{tabular}{lccccc}
\hline & $\mathrm{n}$ & Total & MMF & Control & P-value \\
\hline Donor age (years) & 75 & $59.6 \pm 19.0$ & $59.6 \pm 19.8$ & $59.7 \pm 18.1$ & $0.97^{\mathrm{a}}$ \\
Postmortem time (h) & 80 & $17.0 \pm 15.7$ & $15.5 \pm 11.5$ & $19.1 \pm 20.2$ & $0.37^{\mathrm{a}}$ \\
Preoperative graft endothelial cell density (cells $\left./ \mathrm{mm}^{2}\right)$ & 59 & $2259 \pm 809$ & $2091 \pm 964$ & $2445 \pm 551$ & $0.09^{\mathrm{a}}$ \\
Organ culture period (days) & 78 & $17.9 \pm 7.6$ & $17.8 \pm 8.1$ & $18.1 \pm 6.9$ & $0.96^{\mathrm{a}}$ \\
Graft diameter ( $\leqslant 7.7 />7.7)$ & 86 & $57 / 29$ & $34 / 15$ & $23 / 14$ & $0.48^{\mathrm{b}}$ \\
HLA mismatches (A/B/DR) 0-2/3-6 & 23 & $10 / 13$ & $5 / 9$ & $5 / 4$ & $0.35^{\mathrm{b}}$ \\
\hline
\end{tabular}

\section{${ }^{\mathrm{a} A N O V A .}$}

${ }^{\mathrm{b}} \chi^{2}$-test.

Main criteria regarding efficacy were the occurrence of immune reactions and clear graft survival. Immune reactions were diagnosed by the presence of endothelial precipitates with or without stromal oedema (endothelial immune reaction) or by the presence of non-infectious subepithelial stromal infiltration (stromal immune reaction)..$^{2,25,26}$ Patients with immune reactions were treated with prednisolone acetate $1 \%$ eye drops hourly, and, in severe cases, with fluocortolone $1 \mathrm{mg} / \mathrm{kg}$ body weight per day, tapered over 3 weeks.
Grafts were defined as clear when no opacity was detectable in the central 3-mm zone.

\section{Statistical analysis}

ANOVA was used to compare the metric data of the treatment groups (patient age, follow-up, donor age, postmortem time, preoperative graft endothelial cell density, and organ culture period), and the $\chi^{2}$-test was used regarding gender, classification of risk 
keratoplasties, graft diameter, and HLA data. Clear graft survival and rejection-free interval were estimated using the Kaplan-Meier method. ${ }^{27}$ Statistical significance was determined using the log-rank test. A $P$-value below 0.05 was considered to indicate statistical significance.

All statistical evaluation was carried out using SPSS 12.0 (SPSS Inc., Chicago, IL, USA).

\section{Results}

\section{Demography}

In all, 98 of 140 scheduled patients were included in the study. Fifty-seven were randomised into the MMF group and 41 into the control group. Study recruitment was stopped prematurely due to a statistically significant result from the interim evaluation once $2 / 3$ of the patients had been recruited.

Eleven patients withdrew from the study (seven patients in the MMF group, four in the control group, nine due to protocol deviation, two due to MMF side effects, Table 1). Thus, 87 patients were ultimately considered in the evaluation of efficacy (per-protocol analysis). All MMF-treated patients were included in the safety evaluation. Duration of follow-up was $34.9 \pm 16.3$ months (mean $\pm \mathrm{SD}$ ).

No statistically significant differences appeared between the two patient groups in terms of gender, patient age, follow-up time, indication for surgery (for the above mentioned categories), donor age, postmortem time, preoperative graft endothelial cell density, organ culture period, graft-diameter, and HLA matching (Tables 1 and 2).

A statistically significant difference was found between those diagnoses, which had led to graft failure in the repeat keratoplasty subgroup. Twelve patients in the MMF group and only one in the control group suffered graft failure due to rejection. Seven patients in the MMF group and none in the control group had graft failure due to glaucoma (Table 1).

There was no statistically significant difference in the number of grafts per patient between groups (Table 1). The majority of patients had first repeat keratoplasty and a second graft, respectively. Five patients in the MMF group and only one in the control group had a third graft. One of the MMF group patients had a fourth graft.

\section{Efficacy}

In all, 20 of 87 patients experienced immune reactions -8 of the 50 MMF-treated patients and 12 of the 37 control group patients (Table 3 ). The immune reactions of the MMF group were mostly reversible; 7 of 12 graft rejections of the control group were not. Three of the
Table 3 Efficacy data (per-protocol analysis $(n=87))$

\begin{tabular}{lccc}
\hline End points & MMF $(\mathrm{n}=50)$ & Control (n=37) & P-value \\
\hline Graft rejection & 8 & 12 & $0.044^{\mathrm{a}}$ \\
$\quad$ Acute endothelial & 5 & 9 & \\
Acute stromal & 0 & 1 & \\
Chronic endothelial & 3 & 2 & \\
Reversible & 6 & 5 & \\
Irreversible & 2 & 7 & \\
Graft failure & 10 & 9 & \\
& & & \\
Cause & & & \\
$\quad$ Rejection & 2 & 7 & \\
Glaucoma & 4 & 1 & \\
Epithelial problems & 3 & 1 & \\
Unknown & 1 & - & \\
\hline
\end{tabular}

${ }^{a}$ Log-rank test.

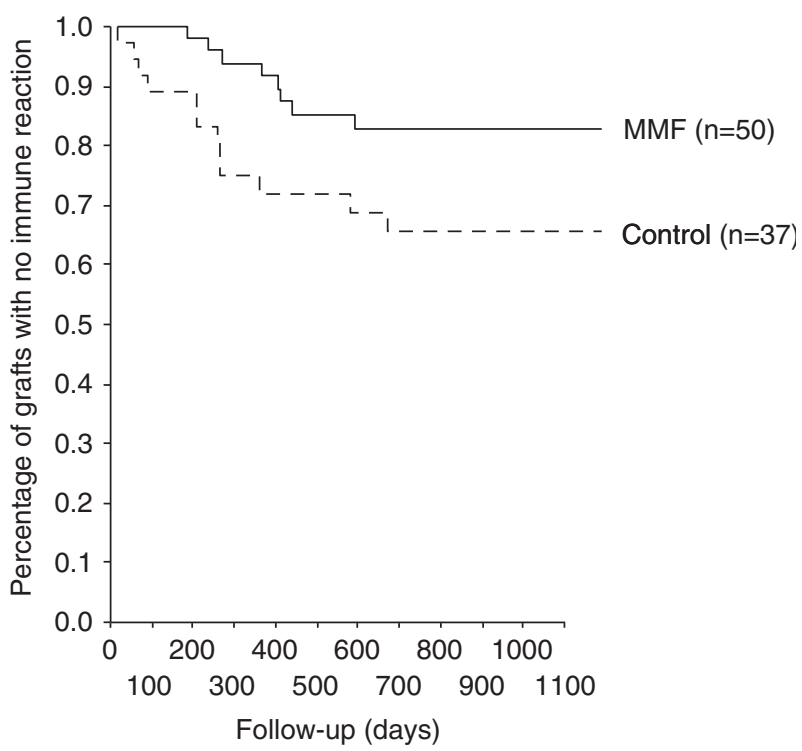

Figure 1 Per-protocol analysis $(N=87)$ : Kaplan-Meier survival plot of immune reaction-free graft survival. MMF: mycophenolate mofetil. Log-rank test: $P=0.044$.

control group patients had two episodes of graft rejection, leading to the graft failure in two of them.

Rejection-free graft survival is illustrated by a Kaplan-Meier survival plot in Figure 1. Percentage of grafts without immune reaction after the mean follow-up time was estimated as $83 \%$ in the MMF group and $64.5 \%$ in the control group (log-rank test; $P=0.044$ ). All of the graft rejections in the MMF group occurred after cessation of the systemic immunosuppression, in contrast to five of the control group immune reactions, which were observed during the first 6 months.

No statistically significant difference appeared regarding graft failure (10 of 50 MMF-treated patients 
and 9 of 37 control group patients; Table 3). However, the percentage of graft failures due to rejection was considerably higher in the control group.

Although our study design was not intention-to-treat mode, we calculated the rejection-free graft survival by means of Kaplan-Meier survival plot for all 98 patients (Figure 2). This calculation shows a similar result with a $P$-value of 0.035 .

\section{Safety}

A total of 36 of $57 \mathrm{MMF}$-treated patients experienced adverse events or possible systemic side effects (Table 4). Systemic immunosuppression was continued in all patients with infections and in both tumour patients. Infections were successfully treated with antibiotics. One patient suffered from prostate gland and bladder carcinoma, detected 5 months after starting systemic immunosuppression. He underwent surgery. The second patient with a malignant tumour had prostate cancer, detected 3 months after starting treatment with MMF. That patient underwent radiation and chemotherapy.

The MMF dosage had to be reduced due to raised liver enzymes in two patients. Neither experienced any graft complications.

In two patients, systemic immunosuppression was stopped due to side effects: one suffered from gastrointestinal disturbances; the other from asthma, pruritus, and fatigue (Table 4).

Not all of the symptoms and diseases presented in Table 4 are likely to be side effects of systemic

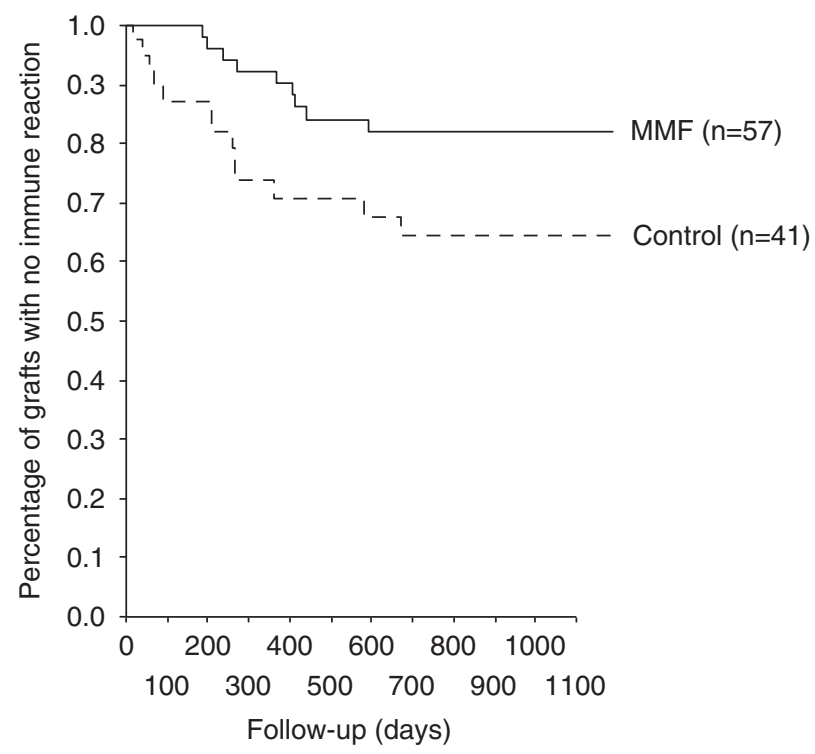

Figure 2 Intention-to-treat analysis $(N=98)$ : Kaplan-Meier survival plot of immune reaction-free graft survival. MMF: mycophenolate mofetil. Log-rank test: $P=0.035$. immunosuppression with MMF. The typical side effects of MMF are infections, leucopaenia, anaemia, and gastrointestinal disturbances.

The control group's adverse events are also presented in Table 4.

\section{Discussion}

The percentage of corneal grafts not revealing an immune reaction following uncomplicated corneal transplantation is over $90 \%$ in some low-risk groups, even without HLA matching or systemic immunosuppression. ${ }^{28,29}$ In spite of this extraordinary success, the rejection rate in high-risk situations, such as repeat keratoplasty, vascularised host cornea, or graft position close to the limbus resembles that in solid organ transplantation, ${ }^{1,30}$ which is why there have been several studies dealing with systemic immunosuppression following high-risk keratoplasty. ${ }^{1,4,5,31-33}$ Yet no prospective, randomised study until now has shown that mid-term systemic immunosuppression actually does prolong rejection-free corneal graft survival in high-risk situations in comparison with a control group without mid-term immunoprophylaxis.

To our knowledge, this is the first prospective, randomised, multicentre study proving the efficacy of systemic immunosuppression with MMF following highrisk keratoplasty.

Only CSA and MMF are currently used routinely in some eye hospitals following high-risk keratoplasty; CSA since the mid-1980s and MMF since 1997.30,31,34 According to our clinical experience, we believed that MMF shows a similar or even superior efficacy in preventing graft rejections, and fewer side effects, than CSA, which is why we carried out this study with MMF. This assumption was nearly confirmed in a retrospective monocentre study. ${ }^{4}$

As corneal transplantation is not a life-saving procedure, long-term systemic immunosuppression is unjustifiable due to the side effects and risk of developing malignancies. The 6.5-month duration of systemic immunosuppression in this study is quite similar to that in earlier studies. . $^{6,32}$

Because of our patient selection, these study results only apply to keratoplasties at a moderately elevated risk of rejection.

We observed no statistically significant differences between the two patient groups in this study in demographic factors, such as gender, patient age, length of follow-up, and so on. Thirteen patients underwent repeat keratoplasty for immunologic graft failure. Twelve of them were in the MMF group and only one in the control group. This is a statistically significant difference (Table 1). We know that repeat keratoplasty following 
Table 4 Adverse events ( $n$ ) in MMF (36 of all treated 57 patients; 63\%) and control groups (14 of all 41 patients; 34\%)

\begin{tabular}{|c|c|c|c|}
\hline \multirow[t]{2}{*}{ Adverse events ${ }^{\mathrm{a}}$} & \multicolumn{2}{|r|}{$M M F$ group } & \multirow{2}{*}{$\begin{array}{c}\text { Control group } \\
\mathrm{n}\end{array}$} \\
\hline & $\mathrm{n}$ & Comments & \\
\hline Arterial hypertension & 17 & & 7 \\
\hline Gastrointestinal disturbances $^{\mathrm{a}}$ & 15 & & 1 \\
\hline Hyperlipidaemia & 11 & & 2 \\
\hline Infections $^{\mathrm{a}}$ & 8 & $\begin{array}{l}\text { Bronchitis, pneumonia, otitis, oral candidiasis, cystitis, } \\
\text { urogenital tract infection, flu, and gastroenteritis }\end{array}$ & - \\
\hline Hyperglycaemia & 7 & & 4 \\
\hline Elevated liver enzymes & 6 & MMF dosage had to be reduced in two patients & 5 \\
\hline Tachycardia & 4 & & - \\
\hline Weight loss & 4 & & - \\
\hline Fatigue & 4 & & - \\
\hline Weight gain & 3 & & - \\
\hline Insomnia & 3 & & - \\
\hline Headache & 3 & & - \\
\hline Malignancies & 2 & $\begin{array}{l}\text { Carcinoma of the prostate gland and the bladder } 5 \text { months } \\
\text { after the beginning of immunosuppression } \\
\text { Carcinoma of the prostate gland } 3 \text { months after the beginning } \\
\text { of immunosuppression }\end{array}$ & - \\
\hline Myalgia & 1 & & - \\
\hline Renal colic & 1 & & - \\
\hline Myocardial infarction & 1 & & - \\
\hline Erythema & 1 & & - \\
\hline Deterioration of atopic eczaema & 1 & & - \\
\hline Muscular cramps & 1 & & - \\
\hline Paresthesia & 1 & & - \\
\hline Ostealgia & 1 & & - \\
\hline Agranulocytosis & 1 & & - \\
\hline Anaemia $^{a}$ & 1 & & - \\
\hline Study withdrawal due to presumed side effects & & & - \\
\hline Gastrointestinal disturbances $^{a}$ & 1 & MMF discontinued after 7 days & - \\
\hline Asthma, pruritus, and fatigue & 1 & MMF discontinued after 5 months & - \\
\hline
\end{tabular}

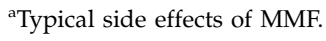

graft failure due to rejection has a higher rejection risk than other repeat keratoplasties. This indicates that the MMF group's rejection risk may have been a priori higher than in the control group, despite randomisation. This imbalance, however, may have introduced a bias towards the control group, and therefore does not compromise the efficacy of MMF.

The same is true for the number of repeat grafts per patient: five MMF group patients and only one control group patient underwent a third graft, and only one MMF group patient had a fourth graft. Although this difference is not statistically significant, the MMF group's rejection risk can be considered higher, as we know that the rejection risk increases with the number of regrafts.

Systemic immunosuppression with MMF showed statistically significant efficacy in reducing immune reactions. As long as MMF was administered, no graft rejection occurred. This finding is compatible with the results of earlier studies, namely that corneal grafts are protected from immune reactions during systemic immunosuppression. ${ }^{4,5}$

Perhaps the most extraordinary finding we made in this study was that a statistically significant difference remained between the two patient groups even in the long run regarding the occurrence of graft rejections (immune reaction-free graft survival after 35 months was $83 \%$ in the MMF group and $64.5 \%$ in the control group). The second interesting fact is that the immune reactions in the MMF group were weaker than those in the control group, as the number of irreversible graft rejections was considerably lower in the MMF-treated patients.

Both of these results might be explained by the development of some kind of immune tolerance for the grafts, induced by mid-term systemic immunosuppression. As MMF intervenes in the immune cascade at a later stage than do other immunosuppressive agents (eg, calcineurin inhibitors 
such as CSA), MMF may be more capable of inducing immune tolerance.

We observed no statistically significant differences in terms of clear graft survival (Table 3 ). However, the percentage of graft opacification due to rejection was considerably higher in the control group. The higher incidence of graft failure due to epithelial problems or glaucoma in the MMF group is probably accidental and not caused by systemic immunosuppression.

The most common adverse events in the MMF group were arterial hypertension, gastrointestinal disturbances, hyperlipidaemia, infections, hyperglycaemia, and elevated liver enzymes. Arterial hypertension, elevated liver enzymes, and hyperglycaemia were the most common adverse events in the control group. It is difficult to define which adverse events are the side effects attributable to MMF, as most current data are based on solid organ transplantation studies with a combination therapy of MMF, CSA, and steroids. Arterial hypertension, hyperglycaemia, and hyperlipidaemia are most likely not the side effects of MMF. ${ }^{35}$

The two malignant tumours may have been coincidental, given the short period between the start of systemic immunosuppression and detection of the tumours. Regarding the known high prevalence of prostate gland carcinoma, the tumours might have been existed and were detected, thanks to the intensive examinations during the study. However, we cannot exclude the possibility that systemic immunosuppression with MMF accelerated the tumour growth. According to some studies in solid organ transplantation, the risk of developing malignancies is not increased with $\mathrm{MMF}^{36,37}$

In conclusion, systemic immunosuppression with $2 \times 1000 \mathrm{mg}$ MMF improved rejection-free graft survival following high-risk keratoplasty statistically significantly even in the long run in this prospective, randomised, multicentre trial. Systemic MMF should be routinely administered following penetrating high-risk keratoplasty, as it is safe and effective in preventing corneal graft rejections.

\section{Acknowledgements}

We thank Roche Pharma AG, Grenzach-Wyhlen, Germany, for financial support of the study. Further participating centres: University Eye Hospital Düsseldorf, Germany; University Eye Hospital, Erlangen, Germany; University Eye Hospital Hamburg, Germany; University Eye Hospital München, Germany; University Eye Hospital Münster, Germany; University Eye Hospital Halle, Germany; University Eye Hospital Homburg/Saar, Germany; University Eye Hospital Essen, Germany.

\section{References}

1 Hill JC. Systemic cyclosporine in high-risk keratoplasty. Short- vs long-term therapy. Ophthalmology 1994; 101: 128-133.

2 Reinhard T, Hutmacher M, Sundmacher R. [Acute and chronic immune reactions after penetrating keratoplasty with normal immune risk]. Klin Monatsbl Augenheilkd 1997; 210: 139-143.

3 Sundmacher R. [Allograft rejection reactions after keratoplasty (author's transl)]. Klin Monatsbl Augenheilkd 1977; 171: 705-722.

4 Birnbaum F, Böhringer D, Sokolovska Y, Sundmacher R, Reinhard T. Immunosuppression with cyclosporine a and mycophenolate mofetil after penetrating high-risk keratoplasty: a retrospective study. Transplantation 2005; 79: 964-968.

5 Birnbaum F, Reis A, Böhringer D, Sokolowska Y, Mayer K, Voiculescu A et al. An open prospective pilot study on the use of rapamycin after penetrating high-risk keratoplasty. Transplantation 2006; 81: 767-772.

6 Reinhard T, Sundmacher R, Godehardt E, Heering P. [Preventive systemic cyclosporin A after keratoplasty at increased risk for immune reactions as the only elevated risk factor]. Ophthalmologe 1997; 94: 496-500.

7 Reinhard T, Reis A, Böhringer D, Malinowski M, Voiculescu A, Heering P et al. Systemic mycophenolate mofetil in comparison with systemic cyclosporin A in high-risk keratoplasty patients: 3 years' results of a randomized prospective clinical trial. Graefes Arch Clin Exp Ophthalmol 2001; 239: 367-372.

8 Reinhard T, Mayweg S, Sokolovska Y, Seitz B, Mittelviefhaus H, Engelmann $\mathrm{K}$ et al. Systemic mycophenolate mofetil avoids immune reactions in penetrating high-risk keratoplasty: preliminary results of an ongoing prospectively randomized multicentre study. Transpl Int 2005; 18: 703-708.

9 Allison AC, Eugui EM. Mycophenolate mofetil and its mechanisms of action. Immunopharmacology 2000; 47: 85-118.

10 Allison AC, Eugui EM. Mechanisms of action of mycophenolate mofetil in preventing acute and chronic allograft rejection. Transplantation 2005; 80: S181-S190.

11 Morris RE, Hoyt EG, Murphy MP, Eugui EM, Allison AC. Mycophenolic acid morpholinoethylester (RS-61443) is a new immunosuppressant that prevents and halts heart allograft rejection by selective inhibition of T- and B-cell purine synthesis. Transplant Proc 1990; 22: 1659-1662.

12 Placebo-controlled study of mycophenolate mofetil combined with cyclosporin and corticosteroids for prevention of acute rejection. European Mycophenolate Mofetil Cooperative Study Group. Lancet 1995; 345: 1321-1325.

13 Mycophenolate mofetil in renal transplantation: 3-year results from the placebo-controlled trial. European Mycophenolate Mofetil Cooperative Study Group. Transplantation 1999; 68: 391-396.

14 A blinded, randomized clinical trial of mycophenolate mofetil for the prevention of acute rejection in cadaveric renal transplantation. The Tricontinental Mycophenolate Mofetil Renal Transplantation Study Group. Transplantation 1996; 61: 1029-1037.

15 Halloran P, Mathew T, Tomlanovich S, Groth C, Hooftman $\mathrm{L}$, Barker C. Mycophenolate mofetil in renal allograft recipients: a pooled efficacy analysis of three randomized, 
double-blind, clinical studies in prevention of rejection. The International Mycophenolate Mofetil Renal Transplant Study Groups. Transplantation 1997; 63: 39-47.

16 Rose ML, Smith J, Dureau G, Keogh A, Kobashigowa J. Mycophenolate mofetil decreases antibody production after cardiac transplantation. J Heart Lung Transplant 2002; 21: 282-285.

17 Wiesner R, Rabkin J, Klintmalm G, McDiarmid S, Langnas A, Punch $\mathrm{J}$ et al. A randomized double-blind comparative study of mycophenolate mofetil and azathioprine in combination with cyclosporine and corticosteroids in primary liver transplant recipients. Liver Transpl 2001; 7: 442-450.

18 Kobashigawa J, Miller L, Renlund D, Mentzer R, Alderman $\mathrm{E}$, Bourge $\mathrm{R}$ et al. A randomized active-controlled trial of mycophenolate mofetil in heart transplant recipients. Mycophenolate Mofetil Investigators. Transplantation 1998; 66: 507-515.

19 Reis A, Reinhard T, Sundmacher R, Braunstein S, Godehardt E. Mycophenolate mofetil and FK506: two novel immunosuppressants in murine corneal transplantation. Transplant Proc 1998; 30: 4344-4347.

20 Reis A, Reinhard T, Sundmacher R, Braunstein C, Godehardt E. Effect of mycophenolate mofetil, cyclosporin $\mathrm{A}$, and both in combination in a murine corneal graft rejection model. Br J Ophthalmol 1998; 82: 700-703.

21 Sundmacher R, Reinhard T. Central corneolimbal transplantation under systemic ciclosporin A cover for severe limbal stem cell insufficiency. Graefes Arch Clin Exp Ophthalmol 1996; 234(Suppl 1): S122-S125.

22 Reinhard T, Sundmacher R. [Perforating keratoplasty in endogenous eczema. An indication for systemic cyclosporin A - a retrospective study of 18 patients]. Klin Monatsbl Augenheilkd 1992; 201: 159-163.

23 Sundmacher R, Stefansson A, Mackensen G. [Postoperative course after keratoplasty]. Fortschr Ophthalmol 1983; 80: 224-227.

24 Hoffmann F. [Suture technique for perforating keratoplasty (author's transl)]. Klin Monatsbl Augenheilkd 1976; 169: 584-590.

25 Alldredge OC, Krachmer JH. Clinical types of corneal transplant rejection. Their manifestations, frequency, preoperative correlates, and treatment. Arch Ophthalmol 1981; 99: 599-604.
26 Pleyer U, Weidle EG, Lisch W, Steuhl KP, Mohrle C, Richter $\mathrm{U}$ et al. [Clinical types of immunologic transplant reactions following perforating keratoplasty]. Fortschr Ophthalmol 1990; 87: 14-19.

27 Kaplan EL, Meier P. Nonparametric estimation from incomplete observations. J Am Stat Assoc 1958; 53: 457-481.

28 Girard LJ, Esnaolo N, Rao R. Allograft rejection after penetrating keratoplasty for keratoconus. Ophthalmic Surg 1993; 24: 40-43.

29 Tompson RW, Price MO, Bowers PJ, Price FW. Long-term graft survival after penetrating keratoplasty. Ophthalmology 2003; 110: 1396-1402.

30 Hill JC. The use of cyclosporine in high-risk keratoplasty. Am J Ophthalmol 1989; 107: 506-510.

31 Reinhard T, Sundmacher R, Heering P. Systemic ciclosporin A in high-risk keratoplasties. Graefes Arch Clin Exp Ophthalmol 1996; 234(Suppl 1): S115-S121.

32 Reis A, Reinhard T, Voiculescu A, Kutkuhn B, Godehardt E, Spelsberg H et al. Mycophenolate mofetil vs cyclosporin A in high risk keratoplasty patients: a prospectively randomised clinical trial. Br J Ophthalmol 1999; 83: 1268-1271.

33 Sundmacher R, Reinhard T, Heering P. Six years' experience with systemic cyclosporin A prophylaxis in high-risk perforating keratoplasty patients. A retrospective study. Ger J Ophthalmol 1992; 1: 432-436.

34 Reinhard T, Reis A, Kutkuhn B, Voiculescu A, Sundmacher R. [Mycophenolate mofetil after penetrating high risk keratoplasty. A pilot study]. Klin Monatsbl Augenheilkd 1999; 215: 201-202.

35 Boots JM, Christiaans MH, van Hooff JP. Effect of immunosuppressive agents on long-term survival of renal transplant recipients: focus on the cardiovascular risk. Drugs 2004; 64: 2047-2073.

36 Lake JR, David KM, Steffen BJ, Chu AH, Gordon RD, Wiesner RH. Addition of MMF to dual immunosuppression does not increase the risk of malignant short-term death after liver transplantation. Am J Transplant 2005; 5: 2961-2967.

37 Robson R, Cecka JM, Opelz G, Budde M, Sacks S. Prospective registry-based observational cohort study of the long-term risk of malignancies in renal transplant patients treated with mycophenolate mofetil. Am J Transplant 2005; 5: 2954-2960. 